\title{
ČEBYŠEV SETS IN HILBERT SPACE
}

\author{
BY \\ EDGAR ASPLUND
}

1. Introduction. A set $T$ in a Hilbert space $H$ is called a Čebyšev set, if for each point $x$ in $H$ there is a unique nearest point $p(x)$ in $T$, i.e.

$$
\|x-y\|>\|x-p(x)\| \quad \text { if } y \in T \text { and } y \neq p(x) .
$$

In this case, we call the function $x \rightarrow p(x)$ the metric projection onto $T$. If a set $T \subset H$ has the property that to each point in $H$ there is a (not necessarily unique) nearest point in $H$, then we will call $T$ an existence set. Clearly, each Čebyšev set is an existence set and each existence set is norm closed. Conversely, if $T$ is boundedly compact, i.e. intersects each bounded and closed subset of $H$ in a compact set, hence in particular if $T$ is any closed subset of a finite-dimensional Hilbert space, then $T$ is an existence set, and so is every closed subset of $T$.

One of the most basic and elementary statements in Hilbert space theory is the theorem that every closed convex set is a Čebyšev set. In 1935, Motzkin showed that if $H$ is finite dimensional then there are no other Čebyšev sets. For infinitedimensional Hilbert spaces the question of the existence of nonconvex Čebyšev sets is still open. Various authors have proved the convexity of Čebyšev sets under additional assumptions [6], [7] (also in more general Banach space settings) and Klee [8] has adduced plausible evidence to support the conjecture that there exist nonconvex Čebyšev sets.

Here we will exhibit two new additional assumptions insuring that a Čebyšev set will be convex. We believe that the second one is the only known such assumption that does not explicitly mention topological properties in some form, only existence properties.

2. A convex function which is the "indefinite integral" of the metric projection. In the following, $K$ will denote some nonconvex Čebyšev set. We thus make explicitly the hypothesis that such exist, and our convexity proofs will be by contradiction. Consider the function

$$
\begin{aligned}
f(x) & =\sup \left\{\langle x, y\rangle-\|y\|^{2} / 2 \mid y \in K\right\} \\
& =\|x\|^{2} / 2-\inf \left\{\|x-y\|^{2} / 2 \mid y \in K\right\} .
\end{aligned}
$$

This is a convex function, since it is the supremum of a set of linear functions, and it is continuous since $f(x) \leqq\|x\|^{2} / 2$, with strict inequality outside $K$ (recall that $K$ is necessarily closed). It is evident that the function $f$ is closely connected with the

Received by the editors February 3, 1969. 
metric projection onto $K$. Actually, if $p(x)$ is the nearest point in $K$ to $x$ in $H$, then $p(x)$ is in the subdifferential $\partial f(x)$ of $f$ at $x$, which by definition means that

$$
f(y) \geqq f(x)+\langle y-x, p(x)\rangle \text { for all } y \text { in } H .
$$

This, of course, follows immediately from (1). On the other hand, one can apply the results of [1] to show that $f$ is actually Fréchet differentiable on a dense $G_{\delta}$ subset of $H$. Moreover, the differentiability properties of $f$ at a point $x$ are related to the continuity properties of $p(x)$. The following statement is a consequence of [2, Theorem 3, Corollary 2 and 3], and can also be proved independently rather easily.

Proposition. The metric projection $p(x)$ is continuous from norm to weak topology at $x$ if and only if $f$ is Gâteaux differentiable at $x$, and continuous from norm to norm topology if and only if $f$ is Fréchet differentiable.

The result of [1] thus says that $p$ is norm to norm continuous on a dense $G_{\delta}$ set. On the other hand, we will soon see that $p$ cannot even be norm to weak continuous at all points of $H$ unless $K$ is convex.

3. A continuity criterion of convexity. We consider next the function $f^{*}$ conjugate to $f$, which is given by the relation

$$
\begin{aligned}
f^{*}(x) & =\sup \{\langle x, y\rangle-f(y) \mid y \in H\} \\
& =\sup \left\{\langle x-p(y), y\rangle+\|p(y)\|^{2} / 2 \mid y \in H\right\} \\
& =\|x\|^{2} / 2+\sup \left\{\left(\|p(y)-y\|^{2}-\|x-y\|^{2}\right) / 2 \mid y \in H\right\} .
\end{aligned}
$$

From the general theory of conjugate convex functions in Hilbert space (cf. Brøndsted [3]) we find that $f^{*}$ is the supremum of all convex functions that are minorants to $\|x\|^{2} / 2$ on $K$. Its epigraph, i.e. the set

$$
\left\{(x, a) \in H \times R \mid a \geqq f^{*}(x)\right\}
$$

is precisely the closed convex hull of the set

$$
\left\{(x, a) \mid x \in K, a \geqq\|x\|^{2} / 2\right\} .
$$

Thus, $f^{*}(x)=+\infty$ for $x$ outside the closed convex hull of $K$. On the other hand, $\operatorname{dom} f^{*}$, i.e. the set on which $f^{*}$ assumes finite values contains $K$, since $f^{*}(x)=f(x)$ $=\|x\|^{2} / 2$ for all $x$ in $K$. Now $\operatorname{dom} f^{*}$ is a convex set, so by hypothesis it cannot equal $K$. In other words, $\operatorname{dom} f^{*}$ intersects the complement $K^{\prime}$ of $K$, but $K^{\prime}$ is an open set, so by a theorem of Brøndsted and Rockafellar [5], there is a point $y$ in $K^{\prime}$ for which $\partial f^{*}(y)$ is nonempty.

Take a point $z$ in $\partial f^{*}(y)$, by the elementary reciprocity relation $y$ is in $\partial f(z)$. But $\partial f(z)$ also contains the point $p(z)$, which is in $K$ and hence must be different from $y$. Thus $f$ cannot be Gâteaux differentiable at $z$, which leads to our first criterion of convexity: 
THEOREM 1. If the metric projection onto a Cebyšev set in Hilbert space is norm to weak continuous, then the set is convex.

COROLlaRY. If the metric projection onto a Cebyšev set in Hilbert space is norm to norm continuous, then the set is convex.

Actually, Theorem 1 follows from the Corollary since if a metric projection is norm to weak continuous at a point in $H$ then it is also norm to norm continuous at that point, by virtue of the uniform convexity of the norm. The author owes this remark to Professor D. Wulbert.

4. An existence property criterion of convexity. Suppose now that the two points $y$ and $z$ are as constructed above, $y \in \partial f(z) \cap K^{\prime}$. In the graph space $H \times R$, the epigraph (2) of $f^{*}$ is supported by the hyperplane

$$
\left\{\left(x, f^{*}(y)+\langle z, x-y\rangle\right) \mid x \in H\right\}
$$

and the projection of the intersection of the two onto $H$ is precisely the set $\partial f(z)$. By hypothesis, $\partial f(z)$ contains more than one point, but the intersection of $\partial f(z)$ and $K$ is the singleton $\{p(z)\}$. Hence the convex set $\partial f(z)$ has extreme points other than $p(z)$, which are therefore in $K^{\prime}$, and we will henceforth assume that $y$ is such an extreme point of $\partial f(z)$. This means that $\left(y, f^{*}(y)\right)$ is an extreme point of the epigraph of $f^{*}$ and hence, by Milman's converse of the Krein-Milman theorem, that it is a weak limit point of the set (3) (a suitable version of Milman's theorem is $\left[4\right.$, Theorem 1]; note that $f^{*}$ is weakly inf-compact in all directions). Let $W$ be a weak neighborhood of $y$ and consider the weak neighborhood

$$
\{(x, a) \mid x \in W, a<\langle z, x\rangle-f(z)+\varepsilon\}
$$

of $\left(y, f^{*}(y)\right)$. Because of what we just said, there exists a point $x$ in $W \cap K$ such that

$$
\|x\|^{2} / 2<\langle z, x\rangle-f(z)+\varepsilon
$$

i.e., since $\varepsilon>0$ can be chosen arbitrarily,

$$
f(z)=\sup \left\{\langle z, x\rangle-\|x\|^{2} / 2 \mid x \in W \cap K\right\} .
$$

Now if $W \cap K$ were an existence set, then any point $u$ in $W \cap K$ nearest to $z$ would satisfy

$$
\|u-z\|=\|p(z)-z\| \quad\left(=\left(\|z\|^{2}-2 f(z)\right)^{1 / 2}\right)
$$

so by the Čebyšev property of $K, u=p(z)$. In particular, the choice

$$
W=\{x \mid\langle x, p(z)-y\rangle \leqq\langle(p(z)+y) / 2, p(z)-y\rangle\}
$$

proves by contradiction

THEOREM 2. If a set $K$ is a Čebyšev set and each closed half-space intersects $K$ in an existence set, then $K$ is convex. 
Corollary 1 (KLEE [7]). If $K$ is Čebyšev and weakly closed, then $K$ is convex.

COROll ARY 2 (EFIMOV-STEČKIN [6]). If $K$ is a boundedly compact Čebyšev set, then $K$ is convex.

COROllaRy 3 (CF. KLEE [8], FOOTNOTE 3). If $K$ is an approximatively compact Čbyšev set, then $K$ is convex.

The three corollaries have been proved by the above quoted authors for wider classes of Banach spaces as well, but it is not known whether Theorem 2 is valid for these. More complete references to this part of the literature can be found in [9].

5. A direct and geometric proof of the convexity of Cebyšev sets with a continuous metric projection. The following alternate proof of the Corollary of Theorem 1 is inspired by the methods of [7], attributed originally to F. A. Ficken.

We may assume without loss of generality that the origin of $H$ belongs to the convex hull of $K$ (the assumed nonconvex Čebyšev set) but not to $K$ itself. The transformation $x \rightarrow x /\|x\|^{2}$ ("inversion in the unit sphere") transforms $K$ into the bounded set

$$
G=\left\{x /\|x\|^{2} \mid x \in K\right\}
$$

Any closed ball $B(x, r)=\{y \in H \mid\|y-x\| \leqq r\}$ containing $G$ must contain the origin in its interior, i.e. satisfy $\|x\|<r$. For otherwise, by inversion, $K$ would be contained in a closed half-space not containing the origin, against assumption.

Given any $x$ in $H$ there is a minimal ball $B(x, t(x)) \supset G$ such that $B(x, r) \ngtr G$ if $r<t(x)$. The image by the involution $x \rightarrow x /\|x\|^{2}$ of the ball $B(x, t(x))$ is the ball

$$
B\left(-x /\left(t^{2}(x)-\|x\|^{2}\right), t(x) /\left(t^{2}(x)-\|x\|^{2}\right)\right)
$$

which is accordingly a maximal ball with center $-x /\left(t^{2}(x)-\|x\|^{2}\right)$ such that the closure of its exterior contains $K$. Thus this ball intersects $K$ precisely in one point, namely $p\left(-x /\left(t^{2}(x)-\|x\|^{2}\right)\right)$. Therefore, the function

$$
x \rightarrow q(x)=p\left(-x /\left(t^{2}(x)-\|x\|^{2}\right)\right) /\left\|p\left(-x /\left(t^{2}(x)-\|x\|^{2}\right)\right)\right\|^{2}
$$

from $H$ into $G$ has the following property:

$$
\|x-y\|<\|x-q(x)\| \quad \text { if } y \in G \text { and } y \neq q(x) .
$$

Thus $G$ is a set with a "unique farthest point property" corresponding to the Čebyšev property with nearest replaced by farthest. Moreover, it follows from (4), since $K$ is bounded away from 0 and $t$ is obviously continuous (in fact convex and Lipschitz, cf. next section) that if $p$ is norm to norm continuous, then so is $q$, and this can easily be seen to lead to a contradiction.

Namely, it is a short exercise in two-dimensional Euclidean geometry to see that $t$ attains its minimum at a unique point $y$. By the very geometry of the situation $q$ cannot be continuous at $y$, not even if restricted to the closed segment $[y, q(y)]$. 
Thus we have another proof of the Corollary of Theorem 1, and by the remark after that Theorem, of the Theorem itself as well.

6. The question of the existence of "Klee caverns". The preceding construction enables one to show also that if the Hilbert space $H$ contains a nonconvex Čebyšev set of any sort, then it contains also one whose complement is bounded and convex. Since the existence of such utterly nonconvex Čebyšev sets was conjectured by Klee in [8], I propose to call them "Klee caverns". In fact, the set

$$
C=\{x \in H \mid t(x) \geqq t(y)+1\}
$$

is a Klee cavern.

To see this we observe first that

$$
B(x, r) \cap B(z, s) \subset B(\lambda x+(1-\lambda) z, \lambda r+(1-\lambda) s) \text { for } 0 \leqq \lambda \leqq 1
$$

by two-dimensional geometry, so that $t$ is a convex function. Furthermore, if $M$ is the bound of the norm on $G$, then

$$
\|x\| \leqq t(x) \leqq\|x\|+M \text { for all } x \in H .
$$

Thus the complement $C^{\prime}$ of $C$ is an open, nonempty, and bounded convex set. Take now an $x$ in $C^{\prime}$ and consider the function

$$
x \rightarrow b(x)=((t(y)+1) x-(t(y)+1-t(x)) q(x)) / t(x) .
$$

It is clear from the fact that $\|b(x)-q(x)\|=t(y)+1$ and

$$
B(b(x), t(y)+1) \supset B(x, t(x)) \supset G
$$

that $t(b(x))=t(y)+1$, so that $b(x)$ is in $C$. Also

$$
\|x-b(x)\|=t(y)+1-t(x) .
$$

If $z$ were a point in $C$ such that

then

$$
\|x-z\|=\|z-x\| \leqq t(y)+1-t(x)
$$

$$
t(z)=\|z-q(z)\| \leqq\|z-x\|+\|x-q(z)\| \leqq t(y)+1
$$

and equality would occur only if $q(z)=q(x)$ and $z-x$ were a positive multiple of $x-q(x)$, i.e. if $z=b(x)$. Thus

$$
\|x-z\|>\|x-b(x)\| \quad \text { if } x \in C^{\prime}, z \in C \text {, and } z \neq b(x) .
$$

Together with the definition $b(x)=x$ for $x$ in $C$ this shows that $C$ is a Čebyšev set, as asserted.

\section{REFERENCES}

1. E. Asplund, Fréchet differentiability of convex functions, Acta Math. 121 (1968), 31-47.

2. E. Asplund and R. T. Rockafellar, Gradients of convex functions, Trans. Amer. Math. Soc. 139 (1969), 443-468. 
3. A. Brøndsted, Conjugate convex functions in topological vector spaces, Mat.-Fys. Medd. Danske Vid. Selsk. 34 (1964), no. 2.

4. - Milman's theorem for convex functions, Math. Scand. 19 (1966), 5-10.

5. A. Brøndsted and R. T. Rockafellar, On the subdifferentiability of convex functions, Proc. Amer. Math. Soc. 16 (1965), 605-611.

6. N. V. Efimov and S. B. Stečkin, Support properties of sets in Banach spaces and Čebyšev sets, Dokl. Akad. Nauk SSSR 127 (1959), 254-257. (Russian)

7. V. L. Klee, Convexity of Čebyšev sets, Math. Ann. 142 (1961), 292-304.

8. - Remarks on nearest points in normed linear spaces, Proc. Colloquium on Convexity (Copenhagen, 1965), Kobenhavns Univ. Mat. Inst., Copenhagen, 1967, pp. 168-176.

9. I. Singer, Some open problems on best approximation, Séminaire Choquet, Paris, 1967.

\section{UNIVERSITY OF STOCKHOLM,}

STOCKHOLM, SWEDEN 